\title{
Variety, Pattern and Isomorphism
}

\author{
Dave Burraston \\ Creativity and Cognition Studios (CCS) \\ Faculty of Information Technology \\ University of Technology, Sydney \\ Australia \\ dave@noyzelab.com
}

\begin{abstract}
Complex systems such as Cellular Automata (CA) produce global behaviour based on the interactions of simple units (cells). Their evolution is specified by local interaction rules that generate some form of ordered, complex or chaotic behaviour patterns. This wide variety of behaviour represents an important generative tool for the artist. Chaotic behaviour dominates rule space, which has serious implications for the serendipitous use of these systems in artistic endeavour. CA are fascinating objects, producing more pattern than a single human is capable of observing within their own lifetime. Obtaining a variety of pattern for free is thus a challenge to the artist and scientist alike. A fresh insight into measuring behaviour and sampling rule space is presented based on empirical evidence. Insights gained from earlier generative music experiments suggest Ashby's cybernetic quantity of variety as a new way of measuring behaviour. A simple and effective method for creating groups of rules with a broad range of behaviour is demonstrated. Isomorphism between automata and music composition technology is shown to exist in terms of basic modular components. This connection provides a fundamental link between the fields and a familiar point of reference for both artist and scientist.
\end{abstract}

Keywords : cellular automata, cybernetics, generative music, variety, pattern 


\section{Introduction}

Different classes of behaviour that CA produce, whether ordered, complex or chaotic, make them interesting to artists and scientists alike. They are fascinating objects, producing more pattern than a single human is capable of observing within their own lifetime. Within the domain of generative music access to a variety of behaviour is essential. CA have played a key part in generative music for many years [1]. Reflective practice has also been utilised to investigate and describe generative music $[2,3,4]$ and some compositions are freely available on-line at [5]. Schon suggests research on fundamental methods of inquiry and overarching theories are a key part of reflective practice [6]. These methods and theories are the springboards for making sense of new situations. A novel method of measuring behaviour in the one dimensional case is proposed and investigated. The method requires little computational resources and is based on the cybernetic quantity of variety, introduced by W. Ross Ashby [7]. It has been suggested that rule choice is a fundamental process in generative music composition with CA [3]. A new approach to this problem is proposed whereby rule groups of broad behaviour are created by considering state space as a symbolic representation of rule space. This method demonstrates an ability to create reasonable diversity from such a chaotic behaviour space. The approach taken in this paper provides an interesting method of studying CA rule and behaviour spaces in general. Ashby describes isomorphism to mean roughly "similar in pattern" and stresses that "It is a concept of the widest range and of the utmost importance to all who would treat accurately of matters in which 'pattern' plays a part." With this in mind some basic isomorphisms of music technology and cellular automata are introduced to provide a fundamental linking point between the fields.

\section{Cellular Automata}

CA are dynamic systems in which space and time are discrete. They may have a number of dimensions, single linear arrays or two dimensional arrays of cells being the most common forms. The algorithm is a parallel process operating on this array of cells. Each cell can have one of a number of possible states. The simultaneous change of state of each cell is specified by a local transition rule. The local transition rule is applied to a specified neighbourhood around each cell. The patterns produced by these systems were classed by Wolfram with one of four qualitative behaviours [8].

Class 1 : Patterns disappear with time or become fixed.

Class 2 : Patterns evolve to a fixed size with periodic structures cycling through a fixed number of states.

Class 3 : Patterns become chaotic.

Class 4 : Patterns grow into complex forms, exhibiting localized structures moving both spatially and temporally.

Other methods of behaviour classification have been devised, an example of six categories is given in [9]. It is known that it is undecidable to assign a CA to a Wolfram class [10] and there are several behaviour prediction parameters, five of which have been surveyed in [11]. The relatively rare complex behaviour of class 4 was suggested by Langton to occur between order (class 1 and 2) and chaos (class 3), termed the "edge of chaos" [12]. 
Langton produced an example schematic illustrating his view of rule space shown in Fig. 1, with spacetime plots of example 1D CA evolutions for each class added. The spacetime plots represent the cellular space horizontally and time evolving in discrete steps downwards. It is important to note that the boundary in rule space between order and chaos contains complex behaviour within it. This implies that the transition from order to chaos may occur in a discontinuous jump to chaos, or may pass through a region of complex behaviour. Langton also supported and promoted work on the global dynamics of CA [13], which offers a new perspective based on the topology of attractor basins, rule symmetry categories and rule clustering. Here one can compare basin topologies and measures between rules to gain insight into different rule behaviours. Attractor basin topology reflects the dynamics of a $\mathrm{CA}$ rule and can be used as a method of identifying ordered, complex and chaotic behaviour.

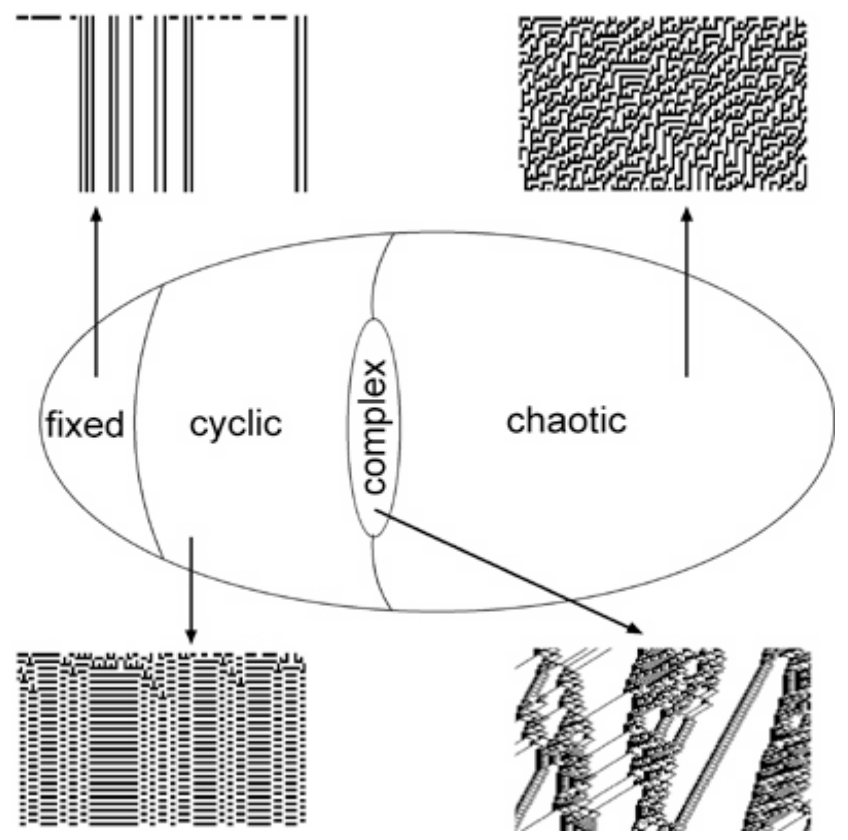

Fig. 1 : Langton's schematic of CA rule space and example spacetime plots of their behaviour

Wuensche's Discrete Dynamics Lab (DDLab) software allows for the exploration of global dynamics [14], as well as many other important aspects of CA and related discrete networks. Wuensche has importantly shown that as the neighbourhood size is increased, the proportion of chaotic rules rises very sharply in a random sampling of rules [15]. This has serious implications for the serendipitous use of these systems in artistic endeavour. The magnitude of the numbers of rules is extremely large, Li has commented on the 5 neighbour rules : "Even if we can produce a spatial-temporal pattern from each rule in 1 second, it is going to take about 138 years to run through all the rules. Considering the redundancy due to equivalence between rules upon 0-to-1 transformations, which cut the time by half, it still requires a solid 69 years." [16]

\begin{tabular}{|c|c|}
\hline Rule type & Total number of rules \\
\hline 2 neighbour & 16 \\
\hline 3 neighbour & 256 \\
\hline 5 neighbour & 4294967296 \\
\hline 7 neighbour & $3.402823669209385 \mathrm{e}+38$ \\
\hline
\end{tabular}

Table 1: Total number of 1D binary CA rules for small neighbourhoods 
The total number of CA rules is a function of the number of states and the size of the neighbourhood. Table 1 . shows a summary of the total number of rules for 1D binary CA with small neighbourhoods. As the neighbourhood is increased there is astronomic increase in the total number of rules. A thorough account of all behaviour types, automatic classification by various parameters and example rules is given in [15], with particular emphasis on complex behaviour in [17]. For a deeper understanding of these concepts the reader is strongly advised to study the literature, in particular Wuensche and Lesser's book, now freely available on the internet [13].

\section{Behaviour Measurement with Cybernetic Variety}

A reasonably universal process for experimenting with generative music production by CA was suggested in [3]. Mathematica [18] can be used to generate spacetime evolutions and convert the binary output into decimal format text files. Code for generating data files using Mathematica's built in CellularAutomaton function is given in [3]. This code is quite general and can be used for any size, generations and number of data files. An example visualisation of ordered, complex and chaotic behaviour mapped to music is shown in Fig. 2.
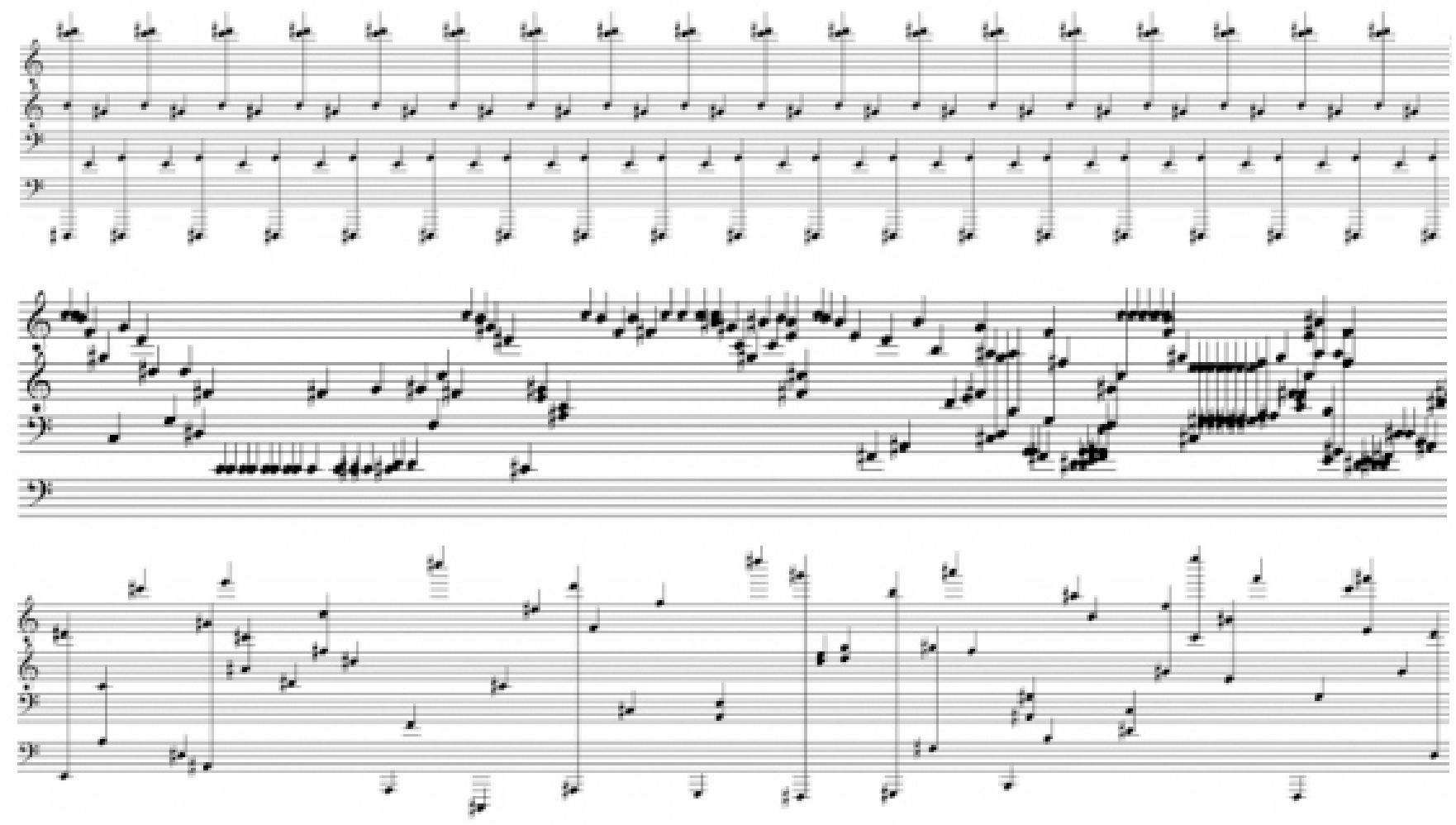

Fig. 2: Musical mappings of ordered (top), complex (middle) and chaotic (bottom) behaviour from large CA.

The mapping process was implemented using AC Toolbox [19] controlling note, velocity and rhythmic timing. A linear conversion was utilized in order to rescale the large data range to a musical range of less than 128 values. The ordered and chaotic sequences appear to show the expected behaviour, but particular attention should be given to the complex note sequence. A deterministic CA will never enter an attractor and then depart from this without external input, such as reseeding or changing the rule in a time dependent manner. This is an important result because it demonstrates that even with extreme scaling each type of behaviour maintains its own identity. The result suggests that measuring and classifying behaviour is achievable from only a small number of 
the total cells in the system. The problem is to find a practical way of measuring this observed phenomena of "quantised" complex behaviour, and also differentiating between order and chaos.

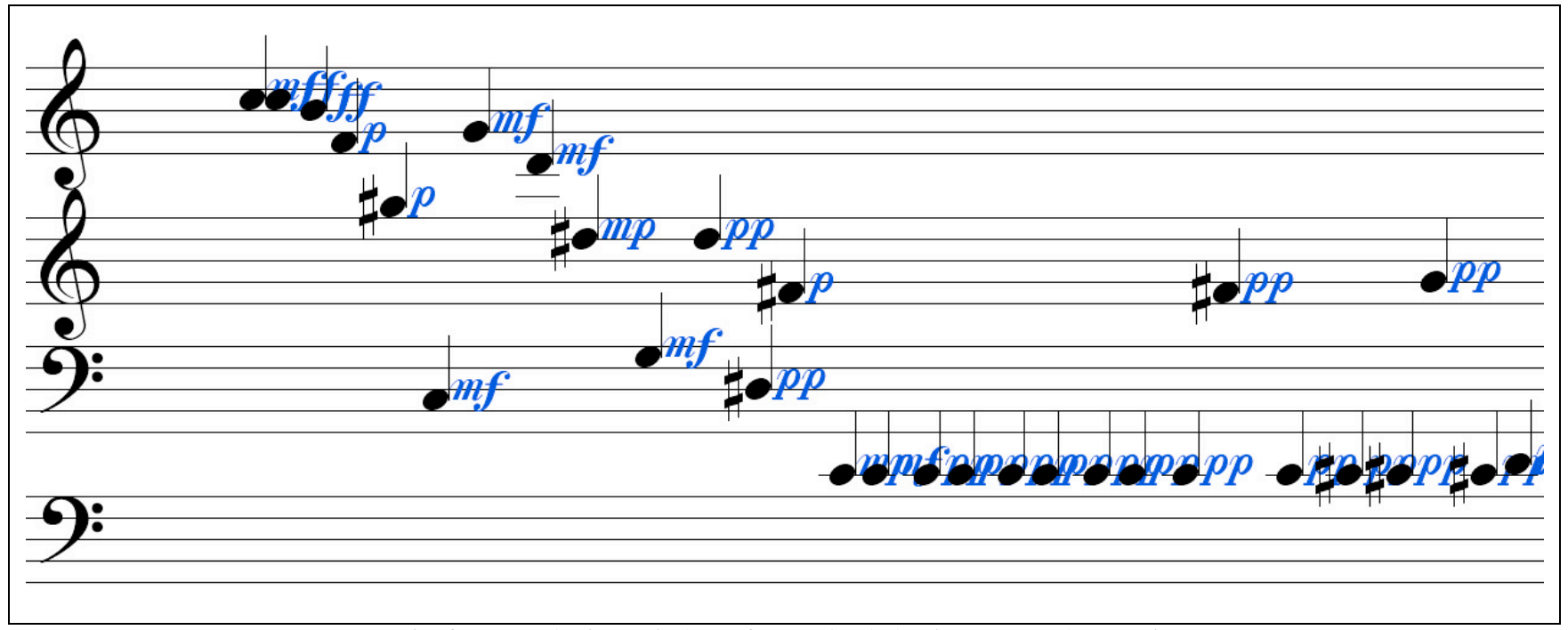

Fig. 3: Example time window of complex behaviour mapped to music

In cybernetics the quantity of variety measures the number of "distinct" elements in a set [7]. For example, given the set $\{\mathrm{a}, \mathrm{b}, \mathrm{d}, \mathrm{g}, \mathrm{a}, \mathrm{a}, \mathrm{d}, \mathrm{b}\}$ which contains 8 elements, there are only 4 distinct elements $\{\mathrm{a}, \mathrm{b}, \mathrm{d}, \mathrm{g}\}$ and the set is thus said to have a variety of 4 . Variety is usually measured in its logarithmic form as $3.322 \log _{10} \mathrm{~N}$ where $\mathrm{N}$ is the number of distinct elements in the set. This allows variety to be expressed in bits and a set with only 1 distinct element, having no variety, will give a measure of zero. Although not explicitly stated in Ashby's account the distinct elements in a set can be simply computed by taking the Intersection of the set with itself. Variety can be expressed in code form very simply, in Mathematica for example it can be expressed :

\section{$3.322 * \log [10$, Length[Intersection[testset, testset]]]}

A section, or time window, of the complex musical mapping is shown in Fig. 3 which if considered as a set of values will have a variety that can be measured. This measure can be applied to the other classes of behaviour and comparisons between measures will be possible. The scaling discussed above has the effect of removing lower significant digits from the data value so the variety measure may be calculated on a small number cells per time window. Three groups of seven cells (equivalent to the neighbourhood size) are measured in this study, taken from the left edge, a third and two thirds across in systems of 150 cells. The size of the time window in the following measurements was chosen as neighbourhood size $x$ neighbourhood size $=49$. Fig. 4 shows ordered (cyclic), complex and chaotic examples of 7 neighbour rules and example variety measures for each. The top plots each show a spacetime evolution of the behaviour, not all the evolutions are shown. The middle plots display left edge temporal variety with bits measure (vertical) against time windows (horizontal). Each time window captured $49 \mathrm{CA}$ time steps and the measure has been performed on 70 windows in total. The bottom plots show variety of left edge and one third sections measured in bits, plotted against each other for all time windows. Plots were also made of the other measures, which were very similar and have been omitted for the sake of brevity. The rule numbers are identified below each plot in hexadecimal. It is clear from the example plots that variety measures can distinguish between the different types of behaviour to a reasonable degree. A summary of comparisons between these behaviour measures is given in Table 2 . 


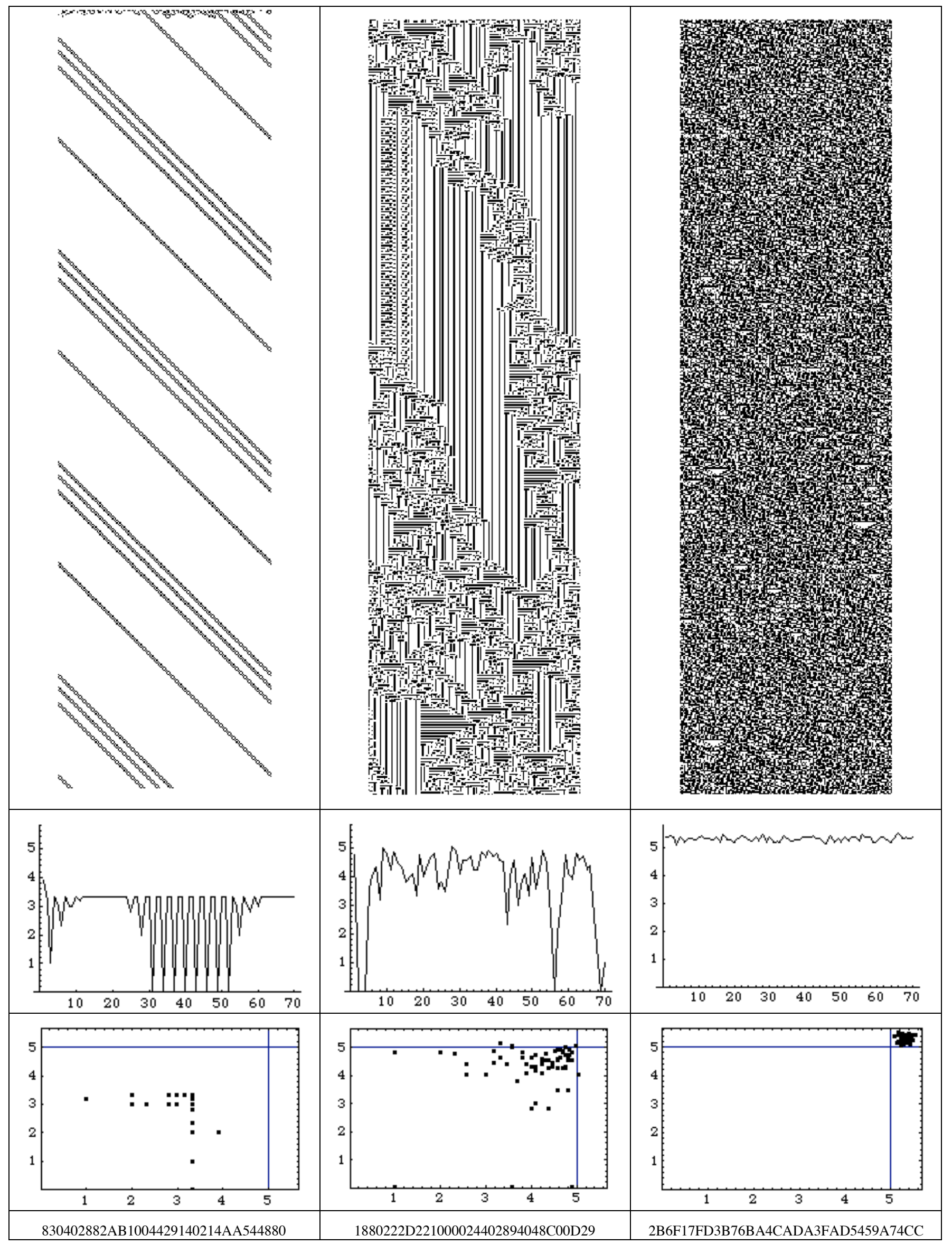

Fig. 4: Spacetime plots with example variety measures of ordered, complex and chaotic behaviour. 


\begin{tabular}{|c|c|c|c|c|}
\hline Behaviour & Ordered (fixed) & Ordered (cyclic) & Complex & Chaotic \\
\hline $\begin{array}{c}\text { Temporal } \\
\text { Variety }\end{array}$ & $\begin{array}{c}\text { Zero or constant } \\
\text { value }\end{array}$ & $\begin{array}{c}\text { Constant values / } \\
\text { Periodic fluctuation }\end{array}$ & $\begin{array}{c}\text { Medium to large } \\
\text { fluctuations }\end{array}$ & $\begin{array}{c}\text { Near maximum } \\
\text { value, very small } \\
\text { fluctuation }\end{array}$ \\
\hline $\begin{array}{c}\text { Variety vs } \\
\text { Variety }\end{array}$ & Very few points & $\begin{array}{c}\text { Small, (sometimes } \\
\text { scattered) cloud of } \\
\text { points }\end{array}$ & $\begin{array}{c}\text { Medium to large } \\
\text { scattered cloud }\end{array}$ & $\begin{array}{c}\text { Small cloud above } \\
5 \text { bits }\end{array}$ \\
\hline
\end{tabular}

Table 2: Comparisons of variety measures observed for CA behaviours

Temporal variety ranges from zero (order), through larger fluctuations (complex) to near maximum values with little fluctuation (chaos). Plotting two or more variety measures against each other gives characteristic scatter plots for each behaviour type. Relatively few points appear for ordered behaviour, due to the rapid evolution to a stable structure. Complex behaviour produces a medium to large sized scattered cloud. Chaotic behaviour produces a small cloud contained in the small boxed area above 5 bits. Empirical evidence suggests that with the settings used the transition to chaos appears around the 5 bit measurement, indicated on the scatter plots.

\section{Pattern for Free?}

Chaotic behaviour dominates rule space, obtaining a variety of pattern is thus a challenge to the artist and scientist alike. A simple method for creating groups of rules with a broad range of behaviour is now presented. If we consider state space to be a symbolic representation of rule space, then a CA spacetime evolution can be interpreted as an example of the self-organisation of rule space. The spacetime output of the generating CA can simply be interpreterd as a list of rules. It follows that this organisation of rule space will reflect the properties of the chosen generating CA for a specific system size. The requirements for a one to one relationship between system size (L) and rule space for small neighbourhood sizes (k) is shown in Table 3.

\begin{tabular}{|c|c|c|c|c|c|c|c|}
\hline Neighbourhood size & 2 & 3 & 4 & 5 & 6 & 7 & $\ldots \mathrm{k}$ \\
\hline System size & 4 & 8 & 16 & 32 & 64 & 128 & $\ldots \mathrm{L}=2^{\wedge} \mathrm{k}$ \\
\hline
\end{tabular}

From Table 3 a system size of $L=128$ is required for a one to one relationship with 7 neighbourhood rule space. Experiments were performed with a number of different rules to sample rule space. A few of the 3 neighbour rules have proved useful, in particular rule 18. An example spacetime evolution of this rule is shown in Fig. 5 for a system size of 128 cells. The top row is a random seed and this is quickly dissolved into a number of triangular structures.

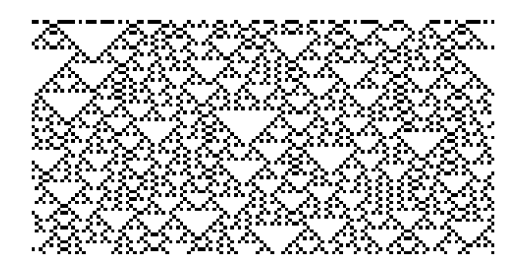

Fig. 5: Spacetime example of the 3 neighbour rule 18

How does a group of rules generated in this way compare to a random selection? Spacetime examples of sixteen randomly chosen 7 neighbour rules are shown in Fig 6. (top), which demonstrate that chaotic behaviour is most commonly found with that method of sampling. Spacetime examples of 7 neighbour rules sampled using rule 18 are shown in Fig 6. (bottom). There is a clear difference between the two groups and a broad range of behaviour can be seen from the rules present in the group generated by rule 18 . 

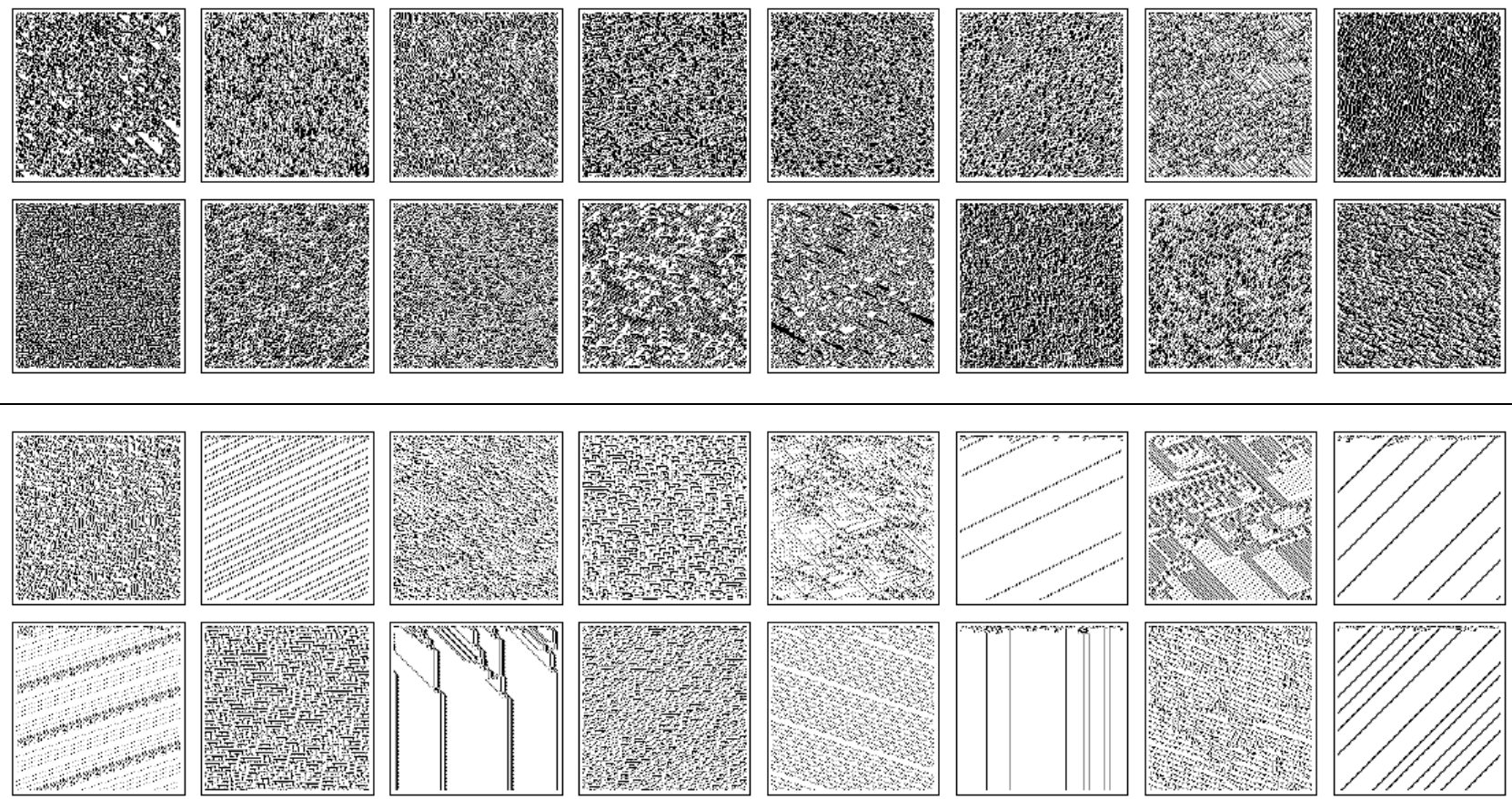

Fig. 6 : Spacetime examples of 7 neighbour rules, randomly generated (top) and generated from rule 18 state space (bottom)

Two other 3 neighbour rules that have proved useful with this method are 22 and 126. Example spacetime patterns for both rules are shown in Fig. 7. where random seeds are again seen to dissolve into triangular structures. Spacetime examples of 7 neighbour rules sampled using rules 22 and 126 are shown in Fig 8.

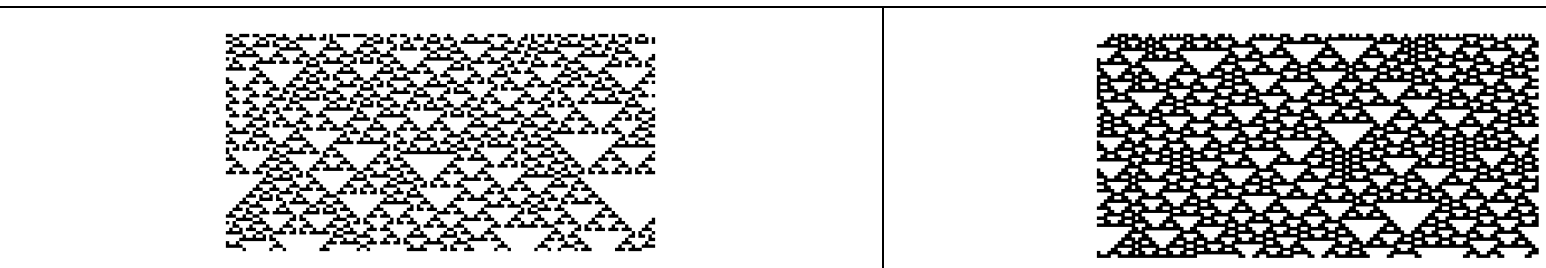

Fig. 7 : Spacetime examples of 3 neighbour rule 22 (left) and rule 126 (right)
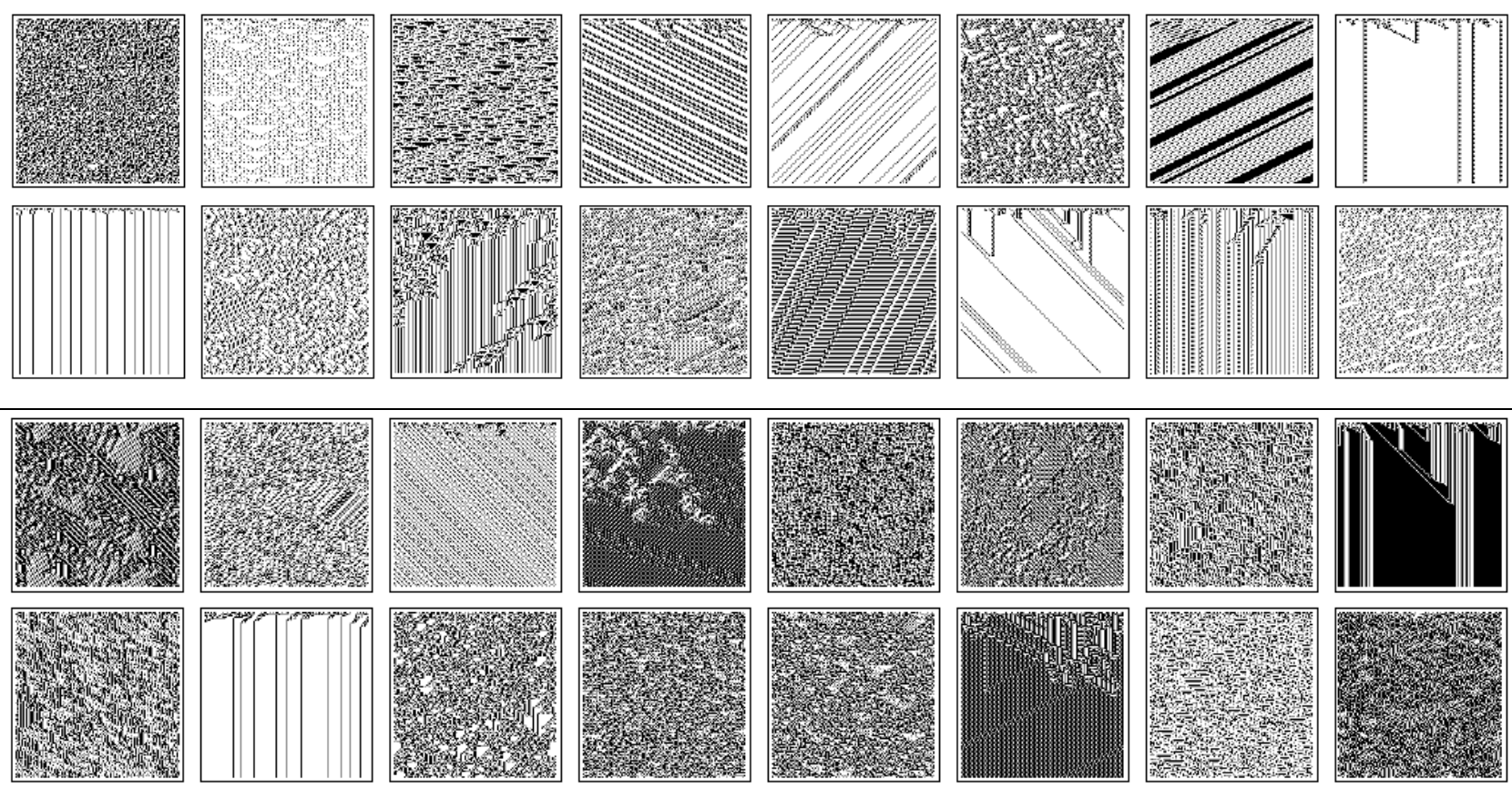

Fig. 8: Spacetime examples of 7 neighbour rules, generated by rule 22 (top) and rule 126 (bottom). 
A wide range of behaviour is also seen from the rules present in these two groups. The generated rule groups shown for rules 18, 22 and 126 are also reasonably typical, based on many experimental runs. Comparing the spacetime patterns of the three generated groups it appears that rule 18 often produces groups with smaller pattern density and very few dark backgrounds. Rule 22 will produce groups having slightly more density and the odd rule with a black background. Rule 126 appears to produce the denser patterns and darker backgrounds. This is interesting as the generating rules themselves also exhibit these features.

These comments are based on empirical observations, the 7 neighbour rule space is extremely large, a microscopic part has been explored. The method has been informally tested with both lower and higher neighbourhood rule spaces where similar results were obtained. The proportion of chaotic rules begins to creep up in the higher neighbourhoods, as is expected, although a deeper investigation will remain the subject of future study. A related and alternative approach to this method using attractor basin topology can be found in [20].

\section{Isomorphism}

Microprocessors have a built in ability to generate nothing with the no operation (NO-OP) instruction. A NO-OP is an instruction which informs the processor to literally do nothing. This very useful ability is known in assembly language, for example to generate timed delay loops within a program. The nearest CA relation to this is the well known 3 neighbour “identity" rule 204, which will always output its initial conditions unchanged. Some example attractor cycles made with Wuensche's DDLab are shown in Fig 9. Ashby defines the identity transform as "An important transformation, apt to be dismissed by the beginner as a nullity" likening it to the opening of a shop keepers cash register at the beginning of the day with the "No Sale" indication. Compare this rule with the well known "buffer" analogue music module and they appear isomorphic. The CA has competitive advantage because with a change of rule becomes a generative pattern module.

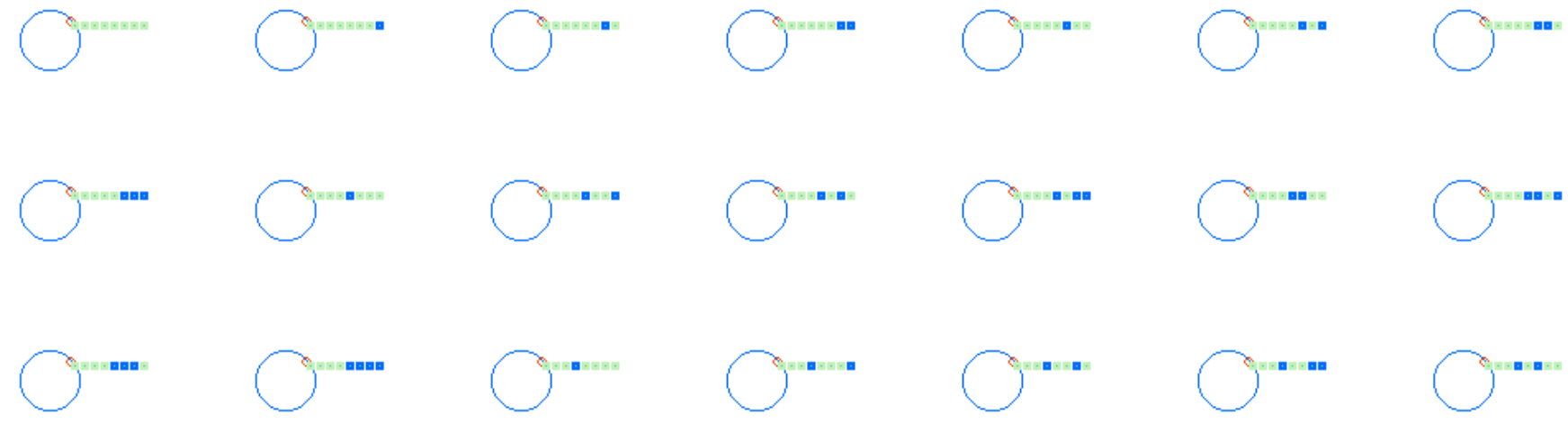

Fig. 9: Example attractor cycles of the identity rule

Another isomorphism with analogue music modules are the equally well known left and right shift rules 170 and 240. Any input pattern will be shifted left (rule 170) or right (rule 240) by one cell per CA step. Analogue sequencer modules commonly have 8,12 or 16 steps and can be stepped sequentially one stage at a time from between 1 and the maximum number of steps available. This operation is from left to right or vice versa. This is analogous to rule 170 and 240 with a single active cell, the attractor is shown in Fig. 10 (top left) and spacetime pattern (top right). Below this are all attractors in an 8 cell system. Three more obvious isomorphisms with music modules are the inverter, noise generator and oscillator. The inverter is equivalent to the "compliment" rule 51 where any state is converted into its inverse. The noise generator and the oscillator's random waveforms are well emulated by the majority of chaotic behaviour in rule space. The oscillator's square waveforms can be provided in two simple ways 1) binary cell output will give a 0 or 1 of varying pulsewidths, 2) attractor 
cycles of all 0's and all 1's will produce a global output of $\min / \max$ value cycles. From these basic isomorphisms we can depart and explore the vast wilderness of pattern, if we have time.

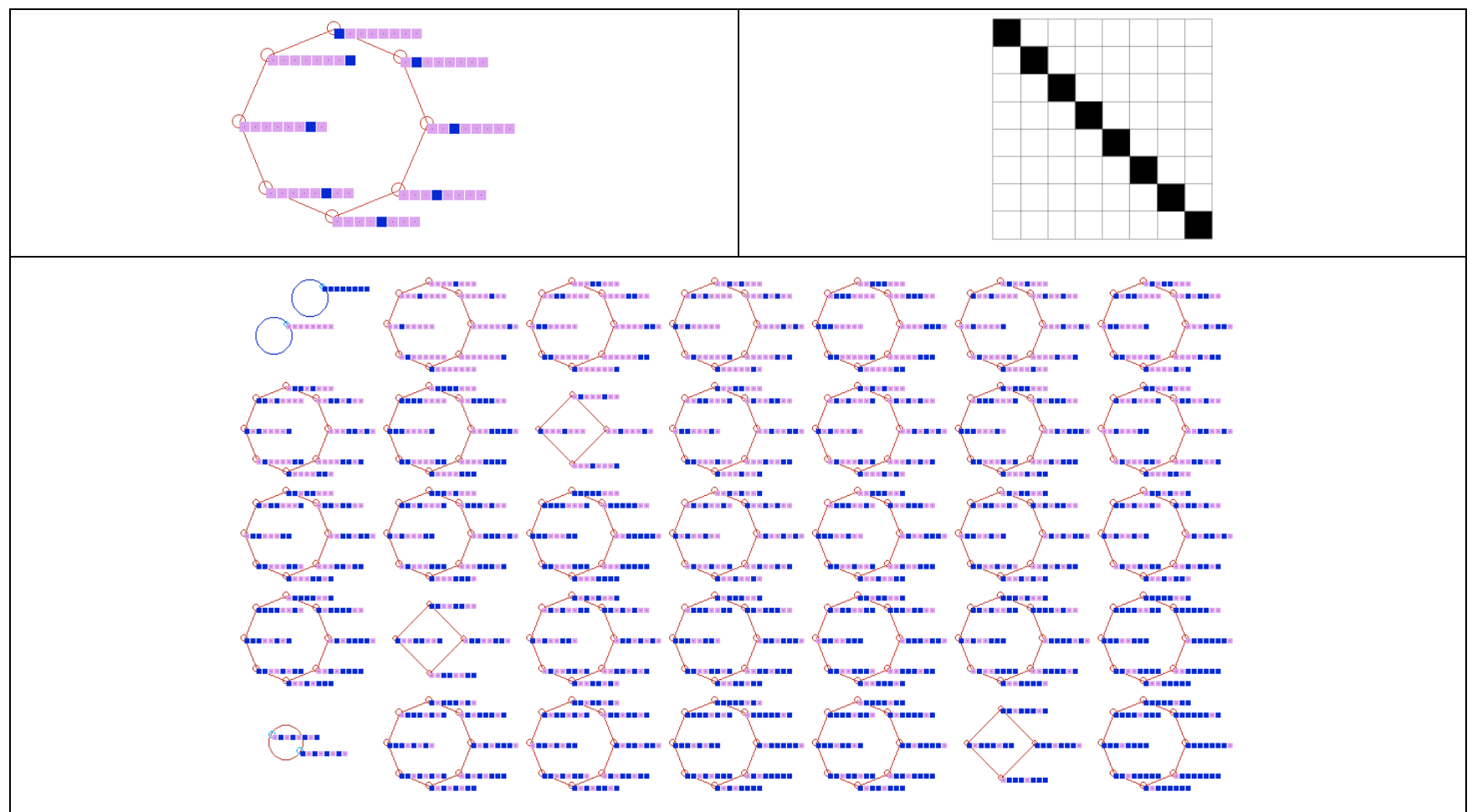

Fig. 10: Single cell attractor (top left) and spacetime pattern of right shift rule (top right). All attractor basins for 8 cells (bottom).

\section{Conclusions}

Generative music experiments have the capability to both produce music and inspire further development of algorithms to assist composition practice. Research based on fundamental methods of inquiry and overarching theory has proved a useful reflective practice methodology. The cybernetic quantity of variety can be applied as a simple and useful measure of behaviour. Computation overhead is minimal and in its simplest form requires only a few cells to obtain a measurement. High variety implies chaos, lower variety order and complex dynamics lie intricately between them. This result supports the notion of order, complexity, chaos as the natural progression of behaviour. There will always be borderline cases, which is also true of other methods of behaviour measurements. Obtaining a variety of pattern for free is a challenge for the artist because of the large proportion of chaotic behaviour. This is not to deny the usefulness of chaos, merely an attempt to restore some balance between order and disorder of potential composition materials. The sampling of rule space can be achieved in more than one way and was demonstrated in the context of rule groupings of varied behaviour. The creation of this algorithmic method of rule selection restores a serendipitous aspect to artistic application. It is perhaps, not necessary to understand the selection (or generation) algorithm in order to make use of it. However, the art constructed by CA and human symbiosis should be viewed as a collaborative process by the human. This philosophy should encourage the artist to understand the automaton, even though the automaton is unlikely to appreciate art. Isomorphism between automata and music composition technology exists in terms of basic modular music components. This connection provides a fundamental link between the fields and a familiar point of reference for both artist and scientist. 


\section{References}

[1] Burraston, D. Edmonds, E. Livingstone, D \& Miranda, E. (2004) Cellular Automata in MIDI based Computer Music. Proceedings of the 2004 International Computer Music Conference.

[2] Burraston, D. \& Edmonds, E. (2004) Global Dynamics Approach to Generative Music Experiments with One Dimensional Cellular Automata. Proceedings of the 2004 Australasian Computer Music Conference.

[3] Burraston, D. (2005) Composition at the Edge of Chaos. Proceedings of the 2005 Australasian Computer Music Conference.

[4] Burraston, D. (2005) One Dimensional Cellular Automata Musical Experiments with Max. Proceedings of the11th International Conference on Human-Computer Interaction. HCI International. (Invited paper)

[5] Burraston, D. (2005) www.noyzelab.com

[6] Schon, D. (2003) The Reflective Practioner. Ashgate Publishing

[7] Ashby, W.R. (1956) An Introduction to Cybernetics. Chapman and Hall.

[8] Wolfram, S. (1984) Universality and Complexity in Cellular Automata. Physica D. 10D, 1-35.

[9] Li, W. Packard, N. H. and Langton, C. G. (1990) Transition Phenomena in Cellular Automata Rule Space. Physica D. 45, 77-94

[10] McIntosh, H. V. (1990) What Has and Hasn't Been Done With Cellular Automata. http://delta.cs.cinvestav.mx/ mcintosh/newweb/marcowhat.html

[11] Oliveira, G., Oliveira, P. and Omar, N. (2001) Definition and Application of a Five-Parameter Characterization of One-Dimensional Cellular Automata Rule Space. Artificial Life 7: 277-301

[12] Langton, C. G. (1991) Life at the Edge of Chaos. In Artificial Life II, Proceedings Vol. X. SFI Studies in the Sciences of Complexity, Addison-Wesley.

[13] Wuensche, A. and Lesser, M. (1992) The Global Dynamics of Cellular Automata : An Atlas of Basin of Attraction Fields of One-Dimensional Cellular Automata. Addison-Wesley. (Freely available as PDF from www.ddlab.com)

[14] Wuensche, A. (2005) The DDLab Manual. Discrete Dynamics Inc. (Freely available as PDF from www.ddlab.com)

[15] Wuensche, A. (1997) Attractor Basins of Discrete Networks. Cognitive Science Research Paper 461, Univ. of Sussex, D.Phil thesis.

[16] Li, W. (1989) Complex Patterns Generated by Next Nearest Neigbors Cellular Automata. Comput. \& Graphics (13)4 pp531-537

[17] Wuensche, A. (1999) Classifying Cellular Automata Automatically: Finding gliders, filtering, and relating space-time patterns, attractor basins, and the $\mathrm{Z}$ parameter, Complexity, Vol.4 no.3, 47-66

[18] Wolfram, S. (2005) Mathematica 5. www.wolfram.com

[19] Berg, P. (2004) Using the ACToolbox. www.koncon.nl/ACToolbox

[20] Burraston, D. (2005) Structuring Cellular Automata Rule Space with Attractor Basins. Proceedings of Generative Arts Practice Symposium 2005 (GAP05). University of technology, Sydney. 